\title{
Labyrinthe
}

$9 \mid 2001$

Numéro 9

\section{Les parents terribles chez Strindberg}

Jeanne Pailler

\section{(2) OpenEdition}

Journals

Édition électronique

URL : http://journals.openedition.org/labyrinthe/927

DOI : $10.4000 /$ labyrinthe.927

ISSN : 1950-6031

Éditeur

Hermann

Édition imprimée

Date de publication : 30 juin 2001

Pagination : 43-58

Référence électronique

Jeanne Pailler, "Les parents terribles chez Strindberg », Labyrinthe [En ligne], 9 | 2001, mis en ligne le 07 juillet 2005, consulté le 23 avril 2019. URL : http://journals.openedition.org/labyrinthe/927 ; DOI : $10.4000 /$ labyrinthe.927

Ce document a été généré automatiquement le 23 avril 2019

Propriété intellectuelle 


\title{
Les parents terribles chez Strindberg
}

\author{
Jeanne Pailler
}

1 Strindberg, dramaturge de la cruauté : la formule de Maurice Gravier ${ }^{1}$ semble particulièrement adéquate s'agissant du théâtre intime de l'écrivain suédois, de celles de ses pièces qui donnent à voir le mécanisme psychologique des relations au sein du couple et de la famille. Ces relations paraissent trop proches, trop intenses pour ne pas mener au déchirement. Elles deviennent ainsi des liens aux sens négatifs du terme : attaches contraignantes qui donnent plus de devoirs que de droits, chaînes pesantes qui appellent à la reconquête d'une liberté, ou bien fil affectif fragile menacé par l'usure du quotidien. La pièce Le Lien (Bandet, 1892 ; c'est là, selon Guy Vogelweith, " le maître mot de la psychologie strindbergienne $\left.{ }^{2} »\right)$ n'est-elle pas l'histoire d'un divorce?

2 La guerre des sexes est chez le dramaturge suédois une thématique récurrente, pour ne pas dire obsessionnelle. Elle s'illustre dans certaines de ses pièces les plus jouées et les plus commentées, entre autres Camarades (Kamraterna, 1886-1887), Le Père (Fadren, 1887), ou La Danse de mort (Dödsdansen, 1900). Nous avons choisi pour notre part de nous intéresser au conflit des générations dans deux pièces courtes, assez mal connues : Devant la mort (Inför Döden) et Amour maternel (Moderskärlek). Dans la première, trois jeunes filles font face à leur père; dans la seconde, une fille est confrontée à sa mère. On a donc affaire à une sorte de diptyque, l'asymétrie des situations et la supériorité numérique du sexe féminin n'étant nullement fortuite - les liens parents-enfants rappellent en effet quelque peu les liens inégaux du couple.

3 Rédigées toutes deux dans le courant de l'année 1892, ces pièces se font suite immédiatement dans l'œuvre de Strindberg et appartiennent au cycle des pièces en un acte rédigées par le dramaturge dans une perspective expérimentale : cherchant «la formule du drame de l'avenir ${ }^{3} »$, il s'efforce d'y condenser au maximum l'action. Condensation paradoxale en apparence, puisque dans les deux pièces qui nous occupent elle va de pair avec une dilatation temporelle liée au récit de l'histoire familiale, qui se réfère aussi bien à l'avant qu'à l'après du drame. 
C'est donc à double titre que la thématique du lien de famille donne lieu à une rénovation formelle chez le dramaturge suédois. D'une part les échanges interpersonnels y sont influencés par la connaissance intime, longuement mûrie, que chacun a de l'autre, jusqu'à lui permettre de s'approprier son discours - ce qui pose la question de l'origine de ce discours. D'autre part, le traitement de la temporalité y est soumis à une dialectique de l'étirement et du resserrement correspondant à l'arrivée de la crise dans l'histoire familiale.

Conflit des générations et lutte des cerveaux

5 Strindberg, dans ses écrits théoriques comme dans ses drames, a marqué à maintes reprises sa fascination pour les travaux de ses contemporains sur les maladies mentales et le fonctionnement du psychisme humain. En particulier, l'unique scène de Devant la mort où Monsieur Durand se trouve face à ses trois filles réunies donne une représentation de ce qu'il appelle le "meurtre psychique ${ }^{4}$ ", c'est-à-dire de la capacité que possède le protagoniste le plus fort d'anéantir en pensée le plus faible. Dans le cas qui nous occupe ici, c'est l'union qui fait la force : Monsieur Durand, tel le roi Lear ${ }^{5}$, est seul face à ses trois filles, qui se trouvent donc en position de supériorité. Adèle, la fille aînée, vient de lui apporter un verre de lait à sa demande, mais Thérèse (la benjamine) le lui retire presque aussitôt, car il n'a pas réussi à trouver l'argent nécessaire pour l'achat du pain quotidien. Allume-t-il une pipe pour se détendre, l'impitoyable jeune fille applique la même mesure punitive :

THÉRÈSE, lui enlevant ses allumettes. Comme cela, au moins, tu ne gaspilleras pas les allumettes ${ }^{6}$.

6 À partir de ce moment, la scène, d'ailleurs très brève, adopte une cadence infernale jusqu'au paroxysme final, celui du meurtre en puissance :

MONSIEUR DURAND [à Thérèse]. [...] je demande simplement que vous

soyez un peu moins cruelles envers moi. Vous trouvez bien de la crème

pour le chat, mais vous refusez du lait à votre père, qui n'a pas mangé

depuis... très longtemps !

THÉRÈSE. C'est donc toi qui as bu jusqu'à la dernière goutte le lait du chat !

MONSIEUR DURAND. Oui, c'est moi.

ANNETTE. Et c'est peut-être lui aussi qui a mangé ce qu'il y avait dans la souricière.

MONSIEUR DURAND. Oui, c'est lui !

ADÈLE. La sale bête !

THÉRÈSE, riant. Et s'il y avait eu du poison dessus?

MONSIEUR DURAND. Tu veux dire : si seulement il y en avait eu !

THÉRÈSE. Et après? Tu as assez dit que tu te tirerais une balle dans la tête ! Il est

vrai que tu ne l'as jamais fait.

MONSIEUR DURAND. « Pourquoi ne t'es-tu pas tiré une balle dans la

tête ?» Voilà au moins qui est franc ${ }^{7}$.

7 La première forme de torture est de l'ordre de l'agir. C'est le geste de privation non seulement des plaisirs les plus simples (fumer) mais surtout des besoins naturels : le lait, aliment vital par excellence, première nourriture prodiguée par la mère, symbolise la régression paradoxale du père au statut de dépendance infantile; le père incapable d'être nourricier devient nourrisson ; cette inversion le met à la merci de ses filles qui, conscientes de leur pouvoir maternant voire matriciel, lui refusent l'existence.

8 Les paroles prennent alors, et très rapidement, le relais des actes. Thérèse formule la première accusation, octroyant encore à son père, par le biais du pronom personnel de deuxième personne " toi ", un statut d'interlocuteur potentiel, de personne dotée d'entendement. Par une gradation dans la cruauté parallèle à la gradation des âges, 
Annette, son aînée de six ans, utilise quant à elle le pronom de troisième personne « lui » ; Monsieur Durand, déjà, est rejeté de l'univers interlocutoire ; psychiquement vaincu, il ne peut que s'adapter au mode d'être impersonnel que lui impose sa fille en reprenant à son compte le pronom de troisième personne. C'est, dans la même logique croissante, la plus âgée des trois filles, Adèle, qui clôturera la progression : "sale bête » est une insulte, donc un acte de langage qui exclut l'échange; et l'on ne s'étonnera pas que cette insulte soit de l'ordre de la déshumanisation, surtout après que Monsieur Durand a reconnu avoir mangé la nourriture destinée aux animaux.

On est donc passé du stade factuel de la torture au stade verbal de la dépersonnalisation (à tous les sens du terme). Reste maintenant le stade mental, le meurtre psychique proprement dit : il est introduit par la question de Thérèse, insinuant la possibilité de la mort du père contenue dans le mot « poison ». On remarquera le processus de suggestion par lequel Monsieur Durand cherche à faire formuler à sa fille l'informulable, «Pourquoi ne t'es-tu pas tiré une balle dans la tête ? ", tandis que celle-ci n'a fait que reproduire cette phrase au discours indirect (« Tu as assez dit que... »), refusant donc de l'assumer. Dès lors apparaît un paradoxe essentiel à la compréhension des rapports personnels chez Strindberg : la conscience du faible suggère ses réponses à la conscience du fort, comme si c'était elle qui souhaitait le meurtre psychique. Le masochisme de Monsieur Durand, son besoin d'entendre les mots qui blessent, voire les mots qui tuent, peut donc apparaître comme le moteur de l'action dramatique.

De l'« intersubjectivité » à l'« intrasubjectivité ${ }^{8}$ »

10 Analysant la scène du Père où le Capitaine pousse Laura à lui dire " disparais ! ", donc à accomplir un meurtre psychique, Peter Szondi remarque que « les paroles de Laura ne sont crédibles que comme la projection des pensées que le Capitaine soupçonne chez sa propre femme..$^{9}$ " On l'a vu, les paroles de Thérèse pourraient, semblablement, être la projection des pensées de Monsieur Durand, et il s'opère dans les relations parentsenfants le même phénomène de «télépathie ${ }^{10}$ » que dans les relations au sein du couple : la conscience du personnage faible pénètre dans celle du personnage fort - du meurtrier psychique - comme pour lui souffler ses pensées. Le combat du fort contre le faible devient lutte intérieure, les personnages apparaissent comme des émanations d'une seule et même conscience déchirée - bref, on n'est plus dans le domaine de l'intersubjectif, mais dans celui de l'intrasubjectif.

Dans Amour maternel, la mère n'est pas seule dans son entreprise de meurtre psychique : le personnage d'Augusta, une habilleuse du théâtre où travaille Hélène (la fille), vient lui prêter main-forte. Cela est particulièrement sensible dans la scène d'ouverture de la pièce où, sur les six répliques qu'elle prononce, cinq sont destinées à provoquer chez la jeune fille un sentiment de culpabilité :

Toujours aussi gentille avec sa mère.

[...]

Preuve que tu as une mère aimante, qui n'a voulu que le bien de son

enfant... Je mens, peut-être?

[...]

Il ne faut pas être impertinente avec sa mère, ma jeune demoiselle [...].

[...]

Tu n'as pas honte, gamine, de répondre comme ça à ta mère?

[...]

Tu veux rappeler à ta mère qu'elle n'a pas d'instruction. Mon Dieu, que

c'est bas ${ }^{11}$ ! 
La seule caractéristique du personnage est la tonalité de reproche contenue dans chacune de ses paroles. Point d'autres données qui permettraient de la construire comme individu distinct : elle n'est qu'un interlocuteur interne à l'esprit d'Hélène, tout entière occupée par son obsession de la faute. Le mot « mère » martèle chaque réplique telle une idée fixe. Et il envahit les dernières paroles par lesquelles Hélène chassera sa demi-sœur Lisen, venue pour tâcher de la délivrer de la tyrannie maternelle, à la fin de la scène IV :

Il ne faut pas dire du mal de mère...

[...]

Sors de cette maison! Rien n'est sacré pour toi, pas même une mère.

$[\ldots]$

Je ne serais pas à mon aise chez des gens qui méprisent ma mère ${ }^{12}$.

Bien plus qu'avec un interlocuteur extérieur, c'est donc avec elle-même qu'Hélène dialogue. Dès lors, la véritable évolution dramatique consiste peut-être dans la décision finale de la jeune fille de reprendre à son compte et d'assumer pleinement les paroles de sujétion qui n'émanaient au départ que d'une partie d'elle-même : le sentiment de culpabilité incarné par Augusta. Semblablement, on a vu comment Monsieur Durand se réappropriait (en les reformulant de manière explicite) les vœux meurtriers de ses filles. Hélène d'Amour Maternel et Monsieur Durand de Devant la mort sont donc plus que des personnages principaux. Ils sont la conscience centrale du «drame subjectif ${ }^{13}$ ».

Le décor, métaphore du psychisme

14 Si le dialogue reflète les diverses voix qui s'expriment au sein de leur intériorité, l'espace, le décor de la pièce, apparaissent eux-mêmes comme une métaphore du psychisme : c'est sur la scène de l'inconscient que se meuvent les protagonistes du drame familial strindbergien. La pension de famille de Monsieur Durand est comme une extension de luimême; le geste suicidaire qu'il accomplit à la fin de la pièce en avalant du poison et le geste incendiaire qui consiste à mettre le feu à la maison sont équivalents, puisqu'il a remplacé par une assurance incendie l'assurance-vie qu'il avait prise : sa propre destruction est donc indissolublement liée à celle du bâtiment où il a habité aux côtés de sa femme puis, après sa mort, de ses seules filles. L'espace extérieur pourrait à la rigueur apparaitre comme une échappatoire à cet emprisonnement mental : la première didascalie de la pièce indique que Monsieur Durand regarde le lac Léman - près duquel est située sa pension - avec des jumelles. Mais ce paysage de neige et d'eau, blanc et glacial, où se détachent des cyprès, est symbole de mort. Sortir de son propre psychisme, c'est-à-dire du système de lutte et d'oppression dans lequel s'est enfermé le personnage, c'est mourir.

Le décor extérieur d'Amour maternel semble au contraire synonyme d'évasion et de naissance possible à une vie sociale et heureuse, loin de l'esclavage auquel Hélène se voit réduite par sa mère : la «station estivale ", la « baie de l'archipel » évoquent les vacances et l'évasion en mer; elles sont aussi les lieux où Hélène peut faire des excursions avec sa demi-sœur Lisen et rencontrer son cousin Gérard, qui est un mari potentiel. Le déplacement infime qui se produit entre la première et la dernière scène reflète tout le processus de la névrose. Les trois mêmes personnages y sont en présence (la mère, la fille, Augusta). Au départ, postée près de la fenêtre, la fille est invitée par sa mère à venir se joindre à la partie de cartes entamée avec Augusta, et refuse ; à la fin de la pièce, elle vient d'elle-même s'asseoir à la table de jeu, s'enfermant entre les murs de son psychisme. Hélène, personnage tragique, est incapable de lutter contre sa dépendance infantile à 
l'autorité. Elle illustre parfaitement, avant la lettre, la comparaison spatiale que Freud effectue entre le cloître et la névrose :

La névrose remplace, à notre époque, le cloître où avaient coutume de se retirer toutes les personnes déçues par la vie ou trop faibles pour la supporter ${ }^{14}$. La temporalité familiale « Une fois retiré l'échafaudage, l'édifice est resté ${ }^{15}$ "

Le drame familial strindbergien s'infléchit vers la mise en scène de la subjectivité du personnage central, vers la mise sur scène d'un psychisme dominé où le processus d'asservissement mis en place par le cerveau dominant fonctionne déjà depuis de nombreuses années. En restant à l'intérieur du décor de la pièce, les personnages principaux de Devant la mort et d'Amour maternel acceptent - métaphoriquement - de demeurer dans l'état de névrose, dont une caractéristique essentielle est la répétition. La temporalité des drames familiaux de Strindberg est donc marquée par le principe du ressassement. Hélène et Monsieur Durand, respectivement la fille opprimée et le père étouffé, avouent leur échec et leur capitulation face à l'action hypnotique exercée sur eux par le cerveau le plus fort, en l'absence même de leur adversaire le plus redoutable : la mère dans Amour maternel, et dans Devant la mort l'épouse défunte, dont la haine de l'époux survit dans le combat mené par les trois filles contre leur père. Monsieur Durand va jusqu'à réclamer à sa fille Thérèse de lui dire " encore une méchanceté ", alors même que tous les mots qu'elle pourra prononcer lui sont connus d'avance ("C'est comme si j'écoutais de la musique, des airs connus du bon vieux temps $\left.!^{16} »\right)$.

17 Le principe du drame subjectif est donc de mettre en lumière la répercussion quotidienne de la lutte du cerveau le plus fort contre le plus faible ; non pas une action, mais la conséquence de cette action. Pour essayer de guérir Hélène de l'opinion négative que la mère de celle-ci lui a donnée de son père, Lisen lui cite l'exemple d'un marin autrefois injustement accusé de vol et dont la mauvaise réputation a survécu à la découverte de son innocence. Elle conclut par cette formule : « une fois retiré l'échafaudage, [...] l'édifice est resté. » Sans le savoir, Lisen énonce l'histoire tragique de sa demi-sœur. Même après que sa mère a, en paroles, libéré Hélène de son esclavage en l'autorisant à aller sans elle chez le directeur de théâtre qui voudrait l'engager, c'est la jeune fille qui refuse : l'habitude névrotique de soumission est plus forte que la prise de conscience provoquée par les révélations concernant son père et le passé de prostituée de sa mère. Le dernier échange de répliques entre les deux jeunes filles dit à la fois la douleur d'Hélène et le « désir d'anéantissement $»$ dont parle Arthur Adamov ${ }^{17}$ :

LISEN. Regarde-moi dans les yeux, avec l'air d'espérer quelque chose de la vie.

HÉLÈNE. Je ne peux pas. Je ne peux pas non plus te remercier pour ta bonne volonté, tu m'as fait beaucoup de mal sans le savoir. Tu m'as réveillée par une piqûre de serpent, moi qui m'étais endormie au soleil, sous les arbres... ${ }^{18}$

Le lien et la chaîne

18 La métaphore du sommeil et de l'éveil employée par Hélène reflète le traitement de la temporalité dans ces pièces courtes de Strindberg. Comme dans la dramaturgie ibsénienne, le temps raconté, celui de l'histoire des personnages, y est bien plus long que celui de la représentation. L'action du drame en elle-même consiste en une révélation : c'est l'éveil du dormeur, qui survient brusquement au terme de conflits ou de processus psychologiques enclenchés depuis très longtemps - vingt ans dans le cas d'Hélène (le temps pour l'enfant d'arriver à l'âge adulte), plus encore dans celui de Monsieur Durand. Mais, quand Ibsen consacre plusieurs actes à l'avènement progressif de la révélation, Strindberg choisit de condenser en un seul acte le temps représenté. Il expose dans un 
texte théorique intitulé Sur le drame moderne et le théâtre moderne sa conception du « drame de l'avenir ", selon laquelle :

Une scène unique, appelée un quart d'heure, semble vouloir devenir le type de pièces qui convient à l'homme moderne ${ }^{19}$.

19 On en arrive ainsi à l'apparent paradoxe d'un temps raconté qui excède de bien loin les limites du temps de la représentation (ou de la lecture de la pièce), tout en étant resserré sous la forme la plus brève possible. C'est que, chez Strindberg, le lien familial est une " chaîne ${ }^{20}$ ». Formée dans la durée (anneau après anneau, année après année), elle se rompt et ne se défait pas : le travail de suppression du lien est nécessairement rapide, intense, voire brutal.

Le principe de la pièce courte comme forme d'accueil de l'histoire familiale permet à l'écrivain suédois de se livrer à une expérimentation qui remet en cause les règles issues de La Poétique d'Aristote, texte fondateur de la dramaturgie en Europe occidentale. La définition aristotélicienne de la tragédie insiste en effet sur le caractère achevé et complet de l'action qu'elle imite ; celle-ci forme un tout, autrement dit, elle " possède un commencement, un milieu et une fin $^{21}$ ». Le début de la pièce - l'exposition, en termes de dramaturgie classique française - est consacré à la présentation des personnages ainsi qu'à celle de leurs desseins et motivations. Le nœud, c'est-à-dire le moment où surgit l'obstacle ou le problème principal qu'il s'agira de résoudre, survient ensuite ; et le dénouement achève l'action en résolvant ledit problème. Ce schéma exige un temps de représentation suffisamment long pour pouvoir se développer. À l'opposé, la pièce en un acte introduit le public in medias res. Ainsi, dans Devant la mort, le nœud est visible dès le commencement de la pièce. L'exposition s'y trouve en effet réduite à un bref échange de répliques entre Monsieur Durand et Adèle, qui suffit à indiquer la situation conflictuelle entre le père et ses trois filles. Aussitôt après survient Pierre, l'employé de la pension : il porte un panier qui contient des factures au lieu de pain. Cette marque de misère est, sur un plan à la fois symbolique et concret, le problème central de la pièce : le père qui n'a pas su trouver l'argent du pain quotidien, qui n'a pas su assumer la fonction nourricière de la mère absente, ce père est indigne de vivre.

Fin immanente et fin imminente ${ }^{22}$

Le public se trouve dès lors face à la chronique d'une mort annoncée. Le dénouement de la pièce courte est en effet connu d'avance : de même que commencement et milieu s'enchevêtrent, commencement, milieu et fin sont presque confondus. On a vu comment Amour maternel superposait, en séquence initiale et en séquence finale, une partie de cartes sur une autre. Ce qui semble le nœud central - l'arrivée de Lisen, la demi-sœur, porteuse de révélations sur le passé familial - n'est qu'une péripétie avortée, un éveil temporaire. La véritable action du drame est cyclique : Hélène, prisonnière de sa névrose, est condamnée à un sort sisyphien. En ce sens, la partie de cartes entamée dans la dernière scène ne clôture pas la pièce, mais l'ouvre au contraire sur la perspective d'un après de la représentation où se reproduira à l'infini la même activité absurde, et où le psychisme dominé réclamera au psychisme dominant sa nourriture quotidienne d'injonctions tyranniques. Ainsi Alice, l'épouse irascible de la Danse de mort, appelle-t-elle le Capitaine à poursuivre sans relâche leur dispute conjugale : "Continuons » est le dernier mot de la pièce - il en pourrait être le premier.

Devant la mort ouvre pourtant une seconde perspective à cette forme moderne du dénouement, qui le fait basculer de la fin immanente à la fin imminente. La fin immanente est celle qui vient d'être évoquée. Cyclique, elle reproduit à la fois le présent 
de la représentation et l'avant du drame ; elle est prévisible. La fin imminente est au contraire celle du suspens, de l'attente d'autant plus intense qu'elle est annoncée comme brève ; ce sont, pourrait-on dire, "les cinq dernières minutes" du quart d'heure dramatique auquel s'est essayé Strindberg. La dernière scène de Devant la mort, qui consiste (comme la première) en un entretien entre Adèle et son père, est marquée par l'avancée de l'incendie dont la fumée remplit peu à peu la pièce. Mais le compte à rebours était enclenché bien avant. Monsieur Durand fait remarquer au tout début de la pièce :

[...] le foehn va se lever bientôt...

Le foehn, c'est-à-dire le vent qui peut propager le feu, est le premier signe avant-coureur de l'incendie. L'imminence devient immédiateté aussitôt après la scène qui oppose le père à ses trois filles réunies, quand le temps change brusquement :

(Le vent se lève, le ciel se couvre. Durand sautant de sa chaise, à Adèle). Éteins vite le fourneau, le foehn arrive ! Monsieur Durand ayant fait - très brièvement - ses adieux à ses filles, et congédié les deux cadettes, il déclare alors à Adèle :

Dans environ cinq minutes, toute la maison brûlera ${ }^{23}$.

On ne saurait mieux souligner l'adéquation entre le temps réel de la représentation et le temps de l'action représentée : le public suit, minute après minute, les derniers instants du personnage, qui sont comme distillés après une vie de souffrances. Strindberg a réalisé magistralement l'objectif qu'il se fixait dans une lettre à Georg Brandes du 29 novembre 1888:

Dans chaque drame il y a une scène ! C'est cette scène que je veux ${ }^{24}$. La trinité conflictuelle

L'écrivain parvient en effet, dans ses deux pièces familiales, à un double effet de condensation. D'une part, le long processus dialogique et interpersonnel par lequel s'est créé peu à peu le lien de dépendance névrotique est retransmis à travers le prisme d'une subjectivité centrale, celle du psychisme dominé ; confronté à l'effet, le lecteur/ spectateur reconstitue les causes. D'autre part, l'histoire familiale est à comprendre sur le plan temporel comme l'irruption d'une crise, aussi aiguë que rapide, au sein d'un quotidien cyclique et répétitif. Que cette crise aboutisse ou non à une transformation radicale - c'est le cas dans Devant la mort, mais non dans Amour maternel - elle fournit la matière centrale de la pièce courte.

27 À ce jeu virtuose de superpositions, il faudrait en ajouter un autre, qui ressortit à la nature même des protagonistes au sein du noyau familial. Le conflit mère/fille n'est compréhensible que s'il dissimule un conflit mère/père ; le conflit père/fille, que s'il recouvre une plaie plus profonde, une chaîne formée à partir de l'union conjugale entre Monsieur et (feu) Madame Durand. De fait, le personnage de l'épouse défunte, dans Devant la mort, et celui du compagnon absent, dans Amour maternel, sont omniprésents dans le discours des personnages. La confrontation de Lisen avec la mère d'Hélène fait ainsi revivre un conflit bien plus ancien, celui du couple : littéralement porte-parole du père, dont elle assume le discours, la demi-sœur de l'héroïne renvoie l'ancienne prostituée à sa culpabilité et à ses mensonges. L'opposition atteint son point culminant dans ces deux répliques antithétiques :

LA FILLE, s'avançant. Il ne faut pas dire du mal de mère...

LISEN. Et comment faire autrement, si je veux dire du bien de mon père.... 
On aperçoit à travers cette lutte des deux sœurs l'éternelle guerre des sexes qui alimente pour ainsi dire toute l'œuvre du Strindberg dramaturge de l'intimité. Le conflit des générations s'efface au profit d'une lutte beaucoup plus ancienne, entamée avant même la naissance des personnages qui l'incarnent dans cette scène. Le lien familial, symbole de déchirement, ne suit pas tant l'axe linéaire de l'ascendance/descendance que celui, simultané, de la collatéralité : la dispute conjugale est reproduite en dispute sororale, et la temporalité s'infléchit décidément vers le cyclique, vers une reproduction à l'infini du même. Conflit des générations, guerre des sexes et lutte des cerveaux se superposent ainsi dans ce que l'on pourrait appeler une trinité conflictuelle, puisque ces trois principes antagonistes, pour finir, n'en font qu'un. La mère d'Hélène « s'effondre » à la fin du réquisitoire que lui adresse, par la voix de sa deuxième fille Lisen, celui qui fut jadis son compagnon : l'on peut considérer que l'élément masculin est le plus fort en l'occurrence, puisqu'il accomplit in absentia le meurtre psychique.

Le rapport de force de Monsieur Durand avec ses filles, s'il est orienté d'emblée par la supériorité numérique du sexe féminin, est aussi à lire en fonction de la domination que feu son épouse peut exercer de manière posthume sur l'esprit d'Adèle, Annette et Thérèse. L'ultime confrontation du protagoniste principal avec sa fille aînée est une tentative de réhabilitation; à chacune des justifications qu'il avance au sujet de sa faillite financière, Adèle rétorque par le même argument, à savoir que la parole d'une morte est irréfutable :

Mère n'a jamais menti.

[...]

Mère disait tout autre chose sur son lit de mort.

[...]

Comme c'est noble d'accuser une morte qui ne peut pas se défendre ${ }^{26 !}$

30 La bataille psychique, ici, est jouée d'avance : on ne lutte pas contre une morte, à moins de n'être plus soi-même de ce monde... et c'est bel et bien la solution que va adopter Monsieur Durand. L'on serait même en droit de se demander si son suicide ne vise pas, en fin de compte, à lui donner la possibilité de continuer, post mortem et ad aeternam, la guerre entamée ici-bas.

31 La thématique du lien de famille est au cœur du questionnement et de l'expérimentation dramaturgiques de Strindberg. La relation parents/enfants, superposée comme on l'a vu à la relation père/mère, est par excellence le lieu où peut se développer le phénomène du meurtre psychique, car celui-ci appelle une force émotive exceptionnelle. Le drame s'insère ainsi dans un contexte quotidien, d'apparence insignifiante, à la faveur d'une exploration des replis les plus profonds d'une conscience individuelle. Le traitement théâtral des rapports interpersonnels cède alors la place à une vision centralisée qui est celle du personnage victime de la domination. La fille d'Amour maternel, le père de Devant la mort se redisent en eux-mêmes les paroles de haine ou les commandements tyranniques qui sont l'aliment indispensable de leur névrose. Cette vision très noire de l'histoire familiale atteint à un paroxysme de cruauté du fait de la condensation extrême de l'intrigue. Sans doute Strindberg, écrivain-alchimiste, a-t-il trouvé dans l'instant de la crise

- celui où l'éternelle guerre des sexes se révèle derrière le conflit des générations - « la formule du drame de l'avenir ». 


\section{BIBLIOGRAPHIE}

ADAMOV Arthur, Strindberg, Paris, éd. de l'Arche, 1955, coll. « Les grands dramaturges ».

ARISTOTE, Poétique, Paris, Les Belles Lettres, 1997, trad. Barbara Gernez.

FREUD Sigmund, Cinq leçons sur la psychanalyse, Paris, Payot, 1966, trad. Yves Le Lay.

GRAVIER Maurice, Théâtre cruel et Théâtre mystique [recueil de textes théoriques de Strindberg], Paris, Gallimard, 1964, trad. Marguerite Diehl.

PAUL Fritz, August Strindberg, Stuttgart, Metzler (Sammlung Metzler $\left.n^{\circ} 178\right), 1979$.

RICCEUR Paul, Temps et récit, vol. II, La Configuration dans le récit de fiction, Paris, Seuil, 1984.

SARRAZAC Jean-Pierre, Théâtres intimes, Arles, Actes Sud, 1989, coll. « Le temps du théâtre ».

STRINDBERG August, Skrifter, vol. XII : Samtidsdramer, Stockholm, éd. Albert Bonniers, 1951 [Inför Döden et Moderskärlek]. - Théâtre complet, Paris, éd. de l'Arche, 1983, vol. III, trad. Tage Aurell pour Devant la mort, trad. Georges Perros pour Amour maternel.

SZONDI Peter, Théorie du drame moderne, Lausanne, L'Âge d'homme, 1983, trad. Patrice Pavis, Jean et Mayotte Bollack.

VOGELWEITH Guy, Psychothéâtre de Strindberg, Paris, Kincksieck, 1972.

\section{NOTES}

*. Cet article s'inscrit dans le cadre d'un travail de recherche mené pour la réalisation d'une thèse de doctorat en Littérature Générale et Comparée sur Le Théâtre de l'insignifiance en Europe autour de 1900, sous la direction de Madame Anne Larue, à l'Université Lumière-Lyon II.

1. Théâtre cruel et Théâtre mystique est le titre d'un recueil d'écrits théoriques de Strindberg publiés sous la direction de M. Gravier (Paris, éd. Gallimard, 1964).

2. Psychothéâtre de Strindberg, Paris, Klincksieck, 1972, p. 99. Guy Vogelweith ajoute (p. 100) : « Pour Strindberg, tout lien affectif avec autrui est une réalité au plus haut point ambiguë. Le lien est à la fois trait d'union et sujétion, promesse d'épanouissement et peur d'étouffer. "

3. Cité dans Théâtre cruel et Théâtre mystique, op. cit., trad. M. Diehl, p. 19.

4. Om själamord (Sur le meurtre psychique) est le titre d'un essai paru en 1887.

5. V. Fritz Paul, August Strindberg, Stuttgart, éd. Metzler (Sammlung Metzler vol. 178), 1979, p. 51.

6. Théâtre complet, Paris, éd. de l'Arche, 1983, vol. 3, trad. Tage Aurell et Georges Perros, p. 57. Ce sera notre édition de référence pour les citations du texte traduit en langue française : «THÉRÈSE (tar undan tändstickorna). Men så ska du åtminstone inte öda på tändstickorna. ", dans Skrifter av August Strindberg, Stockholm, éd. Albert Bonniers, 1951, p. 157 (notre édition de référence pour le texte original en langue suédoise).

7. Théâtre complet, p. 57-58. « DURAND.... jag begär bara att ni äro litet mindre elaka mot mig. Ni har grädde åt katten, men ni unnar inte mjölken åt er far, som inte ätit på... så länge. THÉRÈSE. Det 
är du alltså som inte unnat katten en mjölktår ! DURAND. Ja, det är jag! ANNETTE. Och kanske det är han som ätit opp för råttorna med ? DURAND. Det är han! ADÈLE. Ett sådant svin! [Notons que la traduction française atténue ici la violence de la réplique, qui signifie exactement : « L'espèce de porc ! »] THÉRÈSE (skrattar). Tänk om det hade varit gift på det då ! DURAND. Ack, om det hade varit, menar du! THÉRÈSE. Ja, inte skülle du haft något emot det, som så ofta sladdrat om att du skulle skjuta dig, men integjort det! DURAND. Varför har du inte skjutit dig ? Det är en direkt före bråelse ! ", p. 157.

8. Nous reprenons la formule de Jean-Pierre Sarrazac dans son chapitre consacré à Strindberg, dans Théâtres intimes, Arles, Actes Sud, 1989, coll. « Le temps du théâtre », p. 36-37.

9. Théorie du drame moderne, Lausanne, L'Âge d'homme, 1983, trad. Patrice Pavis, Jean et Mayotte Bollack, p. 36.

10. Jean-Pierre Sarrazac, op. cit, p. 36.

11. Théâtre complet, p. 67-68. " Alltid snäll mot sin mamma!" / "Det visar att Hélène haft en kärleksfull mor, som velat sitt barns bästa... ja, det gör det ! / " Man ska inte vara näsvis mot sin mor, min unga fröken... » / " Vet skäms, din unge, ska du habbla med mor din! » / " Du vill påminna din mor om att hon saknar uppfostran! Fy så simpelt !», Skrifter, p. 163.

12. Théâtre complet, p. 77-78. « Du får inte säga något ont om min mor... » / « Gå ut ur detta hus. Du som icke har något heligt, icke ens moderskapet. » / " Jag skall inte trivas i det sällskapet, som föraktar min mor. ", Skrifter, p. 168.

13. Peter Szondi, Théorie du drame moderne, op. cit., p. 35.

14. «Cinquième leçon », Cinq leçons sur la psychanalyse, Paris, éd. Payot, 1966, trad. Yves Le Lay, p. 61.

15. Théâtre complet, p. 74. « ...när man skulle riva den [...] grunden, befanns buggnaden kvar. », Skrifter, p. 167.

16. Théâtre complet, p. 58. « Det här för mig som att höra musik, kända toner från... den gamla goda tiden... ", Skrifter, p. 157.

17. Strindberg, Paris, éd. de l'Arche, 1955, coll. « Les grands dramaturges », p. 125.

18. Théâtre complet, p. 78-79. «LISEN. Se mig i ögonen och se ut som om du hoppades på livet! DOTTREN. Det kan jag inte! Jag kan inte heller tacka dig för din goda vilja, ty du har gjort mig meront än du vet. Du väckte mig med en orm, när jag slumrat in i en skogsbacke i solskenet... ", Skrifter, p. 157.

19. Théâtre cruel et Théâtre mystique, op. cit., p. 84-85.

20. «Cette chaîne, je la traîne depuis mon enfance ", dit Hélène dans notre traduction de référence (Théâtre complet, p. 72). le texte suédois (Skrifter, p. 165) dit : "Jag från barndomen varit fastsmidd vid denna mor ", c'est-à-dire, littéralement, « J'ai été attachée par le fer [enchaînée] à cette mère depuis mon enfance ».

21. Poétique, Paris, Les Belles Lettres, 1997, trad. Barbara Gernez, p. 29.

22. Nous reprenons la formule de Paul Ricœur dans Temps et récit, Paris, éd. du Seuil, 1984, coll. « Points essais », vol. II, La Configuration dans le récit de fiction, p. 49.

23. Théâtre complet, p. 49, 58 et 60. " Föhn kommer snart att blåsa... » / " (Det har börjat blåsa utanför, och det mulner). DURAND (springer upp ; till Adèle). Släck i spisen! Föhn kommer !» / «Om fem minuter eller så omkring är det nedbrunnet!», Skrifter, p. 153, 157 et 158.

24. Cité dans Théâtre cruel et Théâtre mystique, op. cit., p. 15.

25. Théâtre complet, p. 77. «DOTTREN. Du fär inte säga något ont om min mor... LISEN. Det är omöjligt, när jag skall tala väl om min far... », Skrifter, p. 168.

26. Théâtre complet, p. 61-62. " Min mor ljög aldrig! " / "Nej, så sa icke mor på dödsbädden... » / " Det är ädelt att anklagen en död, som icke kan försvara sig!", Skrifter, p. 158-159. 


\section{RÉSUMÉS}

Influencé par les travaux de ses contemporains sur les maladies mentales et la suggestion, Strindberg fait de la « lutte des cerveaux » un principe structurant de son théâtre du quotidien. Celui-ci se voit investi d'une cruauté particulière lorsqu'il donne à voir le conflit entre parents et enfants, comme c'est le cas dans Devant la mort et Amour maternel. La brièveté de ces deux pièces, qui se composent d'un acte unique, contribue à redéfinir la nature des relations interpersonnelles, en même temps qu'elle permet au dramaturge d'opérer une véritable révolution formelle dans le traitement de la temporalité*.

Family bonds

«Parents terribles » by Strindberg

Due to the influence of the work of contemporary scientists on mental diseases and suggestion, what has come to be termed as "brain struggle" appears as a recurring structure in Strindberg's everyday-life theatre. In Before Death and Maternal Love, the dramatic conflict between parents and children reaches uncommon cruelty. Since both of these short plays are in one act, their density helps the playwright redefine the essence of interpersonal relationships as well as rethink temporality altogether; hence a formal revolution in drama.

\section{AUTEUR}

\section{JEANNE PAILLER}

Jeanne Pailler, qui pratique le théâtre amateur depuis de longues années, termine actuellement une thèse de Littérature générale et comparée sur Le Théâtre de l'insignifiance en Europe autour de 1900, sous la direction d'Anne Larue. À partir d'un corpus d'une quarantaine de pièces d'Henry Becque, d'August Strindberg, d'Oscar Wilde et de Gerhart Hauptmann, sa recherche vise à établir les éléments d'une poétique théâtrale comparée (considérée sous l'angle double du texte et de la représentation), en même temps qu'elle s'interroge sur les sources et les enjeux idéologiques d'une dramaturgie du désengagement politique et de la revendication individualiste. 